\title{
Assessment of meat freshness and spoilage detection utilizing visible to near-infrared spectroscopy
}

Peyvasteh, M., Popov, A., Bykov, A., Meglinski, I.

M. Peyvasteh, A. Popov, A. Bykov, I. Meglinski, "Assessment of meat freshness and spoilage detection utilizing visible to near-infrared spectroscopy," Proc. SPIE 11075, Novel Biophotonics Techniques and Applications V, 110751R (22 July 2019); doi: 10.1117/12.2527194 


\title{
Assessment of meat freshness and spoilage detection utilizing visible to near-infrared spectroscopy
}

\author{
M. Peyvasteh ${ }^{* 1}$, A. Popov ${ }^{1}$, A. Bykov ${ }^{1}$, I. Meglinski ${ }^{1,2,3}$ \\ ${ }^{1}$ Optoelectronics and Measurement Techniques, University of Oulu, Oulu, FI-90014, Finland \\ ${ }^{2}$ National Research Nuclear University "MEPhI", Institute of Engineering Physics for Biomedicine \\ (PhysBio), 115409 Moscow, Russia \\ ${ }^{3}$ Interdisciplinary Laboratory of Biophotonics, National Research Tomsk State University, Tomsk \\ 634050, Russia
}

\begin{abstract}
Visible to near-infrared spectroscopy have been applied for non-invasive assessment of meat freshness. The reduction of oxymyoglobin absorbance associated with freshness drop is clearly seen in the visible range of spectra, as well as supplementary fat, water, and proteins contents variations are observed in the near-infrared range. A table-top spectrophotometer equipped with an integrating sphere was utilized for a shallow probing depth $(80 \mu \mathrm{m})$ and covered 400-1700 nm spectral range. A fiber-optic linear array was coupled to a portable spectrophotometer (measurement range 400-1000 $\mathrm{nm}$ ) for increasing the average probing depth up to $570 \mu \mathrm{m}$. The studied samples of meat experienced an immediate loss of superficial freshness, while kinetics of spoilage was detected after about 2.5 hours. The fiber-optic approach capable for sensing freshness and spoilage process shows promise for design of a compact, portable device for a variety of users at the meat supply chain.
\end{abstract}

Keywords: Spectroscopy, Visible, Near-infrared, Fiber optics, Optical fibers, Meat spoilage, myoglobin, oxymyoglobin

\section{INTRODUCTION}

Meat spoilage is commonly caused by metabolites of contaminant microorganisms. Since spoilage is a subjective judgment by the consumer, it could be influenced by cultural and economic considerations and background as well as by the sensory acuity of the individual and the intensity of the change. Proliferation of bacteria, oxidation and enzymatic autolysis are three basic mechanisms responsible for spoilage of meat ${ }^{1}$.

The traditional methods of meat assessment (sensory evaluation, chemical analysis, and microbiological analysis) are still widely used despite their contact and time-consuming nature is not appropriate for online monitoring of meat production. Optical (spectroscopic) methods are promising compared to other techniques because of their non-contact, fast, real-time, capable for online monitoring, economic and environment-friendly features ${ }^{2}$.

\section{MATERIALS AND METHODS}

Two experimental systems capable to sense different probing depths have been used in this study. As the globally most widely consumed type of meat, pork was chosen to demonstrate the salient features of the spectroscopic approach for detecting loss of freshness and spoilage. A sample was cut to fit a plastic Petri dish $(60 \mathrm{~mm} \times 15 \mathrm{~mm})$ with a rectangular hole to provide direct access of light to the sample and wrapped with a plastic film to prevent water evaporation from the sample surface and was kept at room temperature $\left(23^{\circ} \mathrm{C}\right)$ for $10 \mathrm{~min}$ after taking out from fridge. Although we prepared the samples from the same meat package for each configuration and put them in the same size Petri dish, they were not homogeneous because of slightly different size and level of freshness. However, our aim in this work was to illustrate the differences of the probing depth in the results when using different measurement options. To estimate difference in the penetration depth of the light reflected from the sample for the two different setup configurations, Monte Carlo simulations were performed using a developed online platform (www.biophotonics.fi).

\footnotetext{
${ }^{*}$ Correspondence: motahareh.peyvasteh@oulu.fi
} 


\subsection{Spectrophotometer with an integrating sphere}

In the first configuration, we used an OL 400-LCS lamp source (Optronic Laboratories, USA), a monochromator (OL series 750-M), a highly sensitive detector (OL series 750) and a controller (OL 750-C) connected to a computer.

Two detectors covering two wavelength ranges, 400-1100 nm (Si detector) and 1100-1800 nm (Ge detector), have been used. An integrating sphere enabled acquisition of scattered light from the sample to properly account for absorption. The light spot provided by this setup is $10 \mathrm{~mm}$ in diameter.

\subsection{Fiber-optic linear array}

In another configuration, we used a linear array of eleven optical fibers for sample illumination (1st fiber) and collection of light (11th fiber). The distance between the indicated fibers is $5.3 \mathrm{~mm}$; shorter distances are available using 2nd - 10th fibers. The fibers were stacked with glue and housed in a metal holder. This setup comprises a light source Illuminator EK-1 Fiber Optic Light Source LE.5210-110 (EUROMEX, The Netherlands) with a halogen lamp and a compact CCS200 spectrometer (Thorlabs, USA), both connected to the fiber-optic probe. This setup provided measurements in the 400-1000 nm wavelength range.

\subsection{Monte Carlo simulations}

Here, we use a free online simulation platform ${ }^{3}$ to estimate the sampling volume and the probing depth in each measurement configuration by Monte Carlo method. The optical parameters corresponded to muscle tissue at $632.8 \mathrm{~nm}$ are shown in Table $1^{4}$. This wavelength is chosen for the illustrative purpose. Although the specific light distribution pattern will depend on the wavelength, qualitatively the difference between the discussed configurations will remain.

Table 1. Optical properties of meat (muscle tissue) ${ }^{4}$

\begin{tabular}{|c|c|c|c|c|}
\hline$\lambda, \mathrm{nm}$ & $\mu_{\mathrm{a}}, \mathrm{mm}^{-1}$ & $\mu_{\mathrm{s}}, \mathrm{mm}^{-1}$ & $\mathrm{~g}$ & $\mathrm{n}$ \\
\hline 632.8 & 0.059 & 17.9 & 0.858 & 1.381 \\
\hline
\end{tabular}

\section{Results}

Absorbance changes over time for specific wavelength ranges attributed to the meat different components have been presented in Figure 1, for the integrating sphere configuration in the visible (a) and NIR (b) spectral ranges and the fiberoptic configuration in the visible spectral ranges (c).

(a)

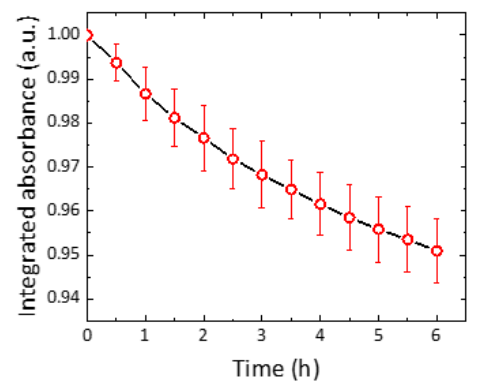

(b)

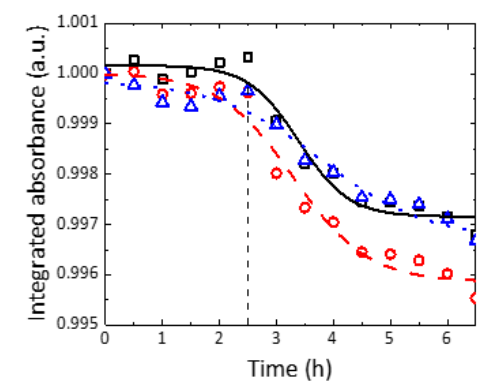

(c)

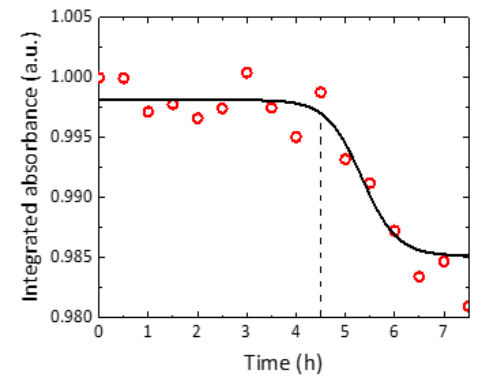

Figure 1. Degradation kinetics of (a) myoglobin, (b) $\mathrm{fat}^{5}$ (1200 nm, triangle/dot), water ${ }^{6}$ (1450 nm, square/solid) and proteins $^{7}$ (1520 and $1600 \mathrm{~nm}$, circle/dash) measured in the integrating sphere configuration and (c) myoglobin measured in. The dashed vertical line in (a) indicates the transition time between the fresh and spoiled pork sample.

The difference between the two configurations is in the probing depth: in the case of the integrating sphere setup, it is shallower due to higher contribution of the surface and subsurface reflected photons. In the second (fiber-optic) configuration, the probing depth is managed through changing the source-detector separation, i.e. by choosing the proper detecting fiber (since the illuminating fiber is the same). Results of the Monte Carlo simulations in Figure 2 illustrate this aspect. 

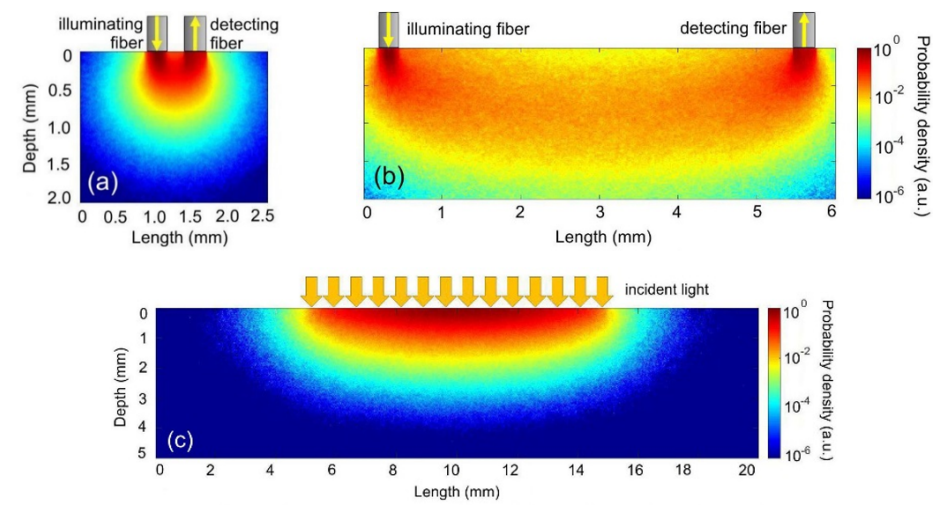

Figure 2. Spatial distributions of the detected signal in the pork sample in the fiber-optic (a and b) and in the integrating sphere (c) configurations assessed by Monte Carlo simulations. The distance between the illuminating and the detecting fibers is $0.53 \mathrm{~mm}$ (a) and $5.3 \mathrm{~mm}(\mathrm{~b})$.

\section{CONCLUSIONS}

According to the results, it is possible to observe the decreasing trend in the light absorbance in both visible and NIR spectral ranges showing loss of freshness and meat spoilage over time. In the visible wavelength range, absorbance changes at $540 \mathrm{~nm}$ and $580 \mathrm{~nm}$ wavelengths allow for monitoring oxymyoglobin content ${ }^{8}$ degradation, which is associated with loss of freshness. In the NIR region, monitoring changes in the absorbance of fat (1200 nm), water (1450 $\mathrm{nm}$ ), and proteins (1525 nm and $1600 \mathrm{~nm}$ ) show promise to detect spoilage. Specifically, absorbance of the superficially located oxymyoglobin decays immediately but only after 4 hours at the depth of $0.57 \mathrm{~mm}$, while absorbance of surface water and protein components experiences a steep decrease only after about 2.5 hours that could be interpreted as a beginning of the spoilage process.

\section{REFERENCES}

[1] Olaoye, O. A. \& Ntuen, I. G. "Spoilage and preservation of meat: a general appraisal and potential of lactic acid bacteria as biological”. IRJOB. 2(1), 33-46 (2011).

[2] Kamruzzaman, M., Makino, Y., \& Oshita, S. "Non-invasive analytical technology for the detection of contamination, adulteration, and authenticity of meat, poultry, and fish: A review" Anal. Chim. Acta. 853(1), 19-29 (2015)

[3] Doronin, A. \& Meglinski, I. "Peer-to-peer Monte Carlo simulation of photon migration in topical applications of biomedical optics" J. Biomed. Opt. 17(9), 90504 (2012).

[4] Bashkatov A. N., Genina E. A. \& Tuchin, V. V. "Optical properties of skin, subcutaneous, and muscle tissues: A review" J. Innov.e Opt. Health Sci. 4(1), 9-38 (2011).

[5] ElMasry, G. \& Nakauchi, S. "Prediction of meat spectral patterns based on optical properties and concentrations of the major constituents" Food Sci. Nutr. 4(2), 269-283 (2016).

[6] Firtha, F., Jasper, A., \& Friedrich, L. "Spectral and hyperspectral inspection of beef ageing state" Presented at Chinese-European cooperation for a long-term sustainability. Budapest, Hungary (2011).

[7] Barbin, D. F., Kaminishikawahara, C. M., \& Soares, A. L. "Prediction of chicken quality attributes by near infrared spectroscopy" Food Chem. 168, 554-580 (2015).

[8] Park, B., Chen, Y. R., Hruschka, W. R., \& Koohmaraie, M. "Principal component regression of NIR reflectance spectra for beef tenderness prediction” ASABE. 44(3), 609-615 (2001) 